\title{
Uso de la Fibrina Rica en Plaquetas Inyectable (i-PRF) en Defectos Infra Óseos en Terapia Periodontal no Quirúrgica. Reporte de Dos Casos
}

\author{
Use of Injectable Platelet-Rich Fibrin (i-PRF) in Infra Osseous Defects \\ in Non-Surgical Periodontal Therapy. Report of Two Cases
}

Correa, A. J. ${ }^{1,2,3}$; Alister, J. P. ${ }^{1}$ \& Manterola, C. ${ }^{1,4,5}$

CORREA, A. J.; ALISTER, J. P. \& MANTEROLA, C. Uso de la fibrina rica en plaquetas inyectable (i-prf) en defectos infra óseos en terapia periodontal no quirúrgica. reporte de dos casos. Int. J. Odontostomat., 13(2):271-274, 2019.

RESUMEN: Entre los concentrados plaquetarios de segunda generación, ha suscitado creciente interés, el uso de fibrina rica en plaquetas y leucocitos inyectable (i-PRF); que se obtiene a partir de la centrifugación inmediata de sangre venosa del propio individuo, y que aporta concentraciones elevadas de factor de crecimiento vascular endotelial, factor de crecimiento transformante beta, y factor de crecimiento derivado de plaquetas, entre otras proteínas que inician y coordinan el proceso reparativo. Su nula citotoxicidad y consistencia líquida abren un nuevo campo de estudio y experimentación en el ámbito de la Cirugía Oral y de la Periodoncia, como sustancia para irrigar. El objetivo de este manuscrito fue reportar el uso del i-PRF como irrigador subgingival en el tratamiento periodontal convencional de defectos infra óseos con 6 meses de seguimiento. En ambos casos, se verificó un efecto positivo de irrigación, lo que abre el debate al uso de productos farmacéuticos tradicionales como la clorhexidina versus preparados autólogos sin efectos adversos reportados a la fecha.

PALABRAS CLAVE: terapia periodontal, i-PRF, defectos infraóseos, irrigación.

\section{INTRODUCCIÓN}

En una población con crecientes expectativas de vida, el aumento en la prevalencia de patologías crónicas y sus secuelas se convierten en un desafío para la Odontología actual en términos de poder revertir estos procesos a fin de mejorar el pronóstico de los dientes remanentes y de las futuras rehabilitaciones a planificar (Tonetti et al., 2017).

La fibrina rica en plaquetas y leucocitos (L-PRF) cuenta ya con suficiente evidencia para transformarse en una alternativa más a considerar al momento de tratar estas secuelas, como por ejemplo: defectos periodontales infra óseos, deficiencias de reborde alveolar, preservación de alveolos post-exodoncia, entre otros (Castro et al., 2017a,b; Nelson et al., 2018).

Para la versión inyectable (i-PRF) se describen sus primeras aplicaciones para favorecer la regenera- ción ósea guiada en conjunto con sustitutos óseos, protocolos conocidos como Sticky Bone o PRF-Block (Sohn, 2010; Cortellini et al., 2018), existiendo ya estudios in-vitro en donde miden su contenido celular de plaquetas y leucocitos y liberación de factores de crecimiento como el VEGF, TGF-ß, PDGF, reportando comportamiento similar al del L-PRF (Pinto et al., 2018; Varela et al., 2018). En condiciones fisiológicas, estos factores son responsables del inicio y regulación de la respuesta reparativa. Sus propiedades quimiotácticas sobre células endoteliales, fibroblastos y otras células de la respuesta inmune hacen de estos concentrados un aporte real al tratamiento de las secuelas producto de las patologías más prevalentes, como la pérdida parcial y/o total del soporte dentario.

A lo anterior se puede agregar la potencial versatilidad de la versión liquida o inyectable del PRF lo

\footnotetext{
${ }^{1}$ Programa de Doctorado en Ciencias Médicas, Universidad de La Frontera, Temuco, Chile.

2 Universidad San Sebastián, Facultad de Odontología, Sede de la Patagonia, Chile.

${ }^{3}$ Hospital Dr. Eduardo Schütz Schroeder, Puerto Montt, Chile.

${ }^{4}$ Departamento de Cirugía y Traumatología, Universidad de La Frontera, Temuco, Chile.

${ }^{5}$ Centro de Excelencia en Estudios Morfológicos y Quirúrgicos (CEMyQ), Universidad de La Frontera, Temuco, Chile.
} 
que eventualmente podría ampliar su rango de uso incluso a procedimientos no quirúrgicos.

El objetivo de este escrito fue reportar el uso del i-PRF como irrigador subgingival en el tratamiento periodontal convencional de defectos infra óseos con 6 meses de seguimiento.

\section{MATERIAL Y MÉTODO}

Caso clínico 1. Paciente sexo femenino, 34 años de edad, sin antecedentes mórbidos. Consultó por trauma de la oclusión en el grupo 2 por sobremordida en MIC, protrusión y lateralidad; observándose movilidad III de Müller en diente 1.2 y presencia de supuración. Al examen clínico y radiográfico se verificó la presencia de un saco periodontal de $10 \mathrm{~mm}$ de profundidad (Figs. 1a y 1b). Se alivió oclusión se procedió a la toma de muestra para i-PRF mediante punción venosa de la vena cubital, tomando $5 \mathrm{ml}$ en 2 tubos Vaccuette (Fig. 2) y centrifugándolo de inmediato a $2700 \mathrm{rpm}$ por 3 min según protocolo descrito (Pinto et al.). Simultáneamente y bajo anestesia local se realizó destartraje supra y subgingival y pulido radicular del diente afectado comprobando la presencia final de una superficie radicular lisa y dura. Terminado el centrifugado se retiró la tapa blanca del tubo y se aspiró con jeringa hipodérmica de $5 \mathrm{ml}$ sólo el contenido superior del tubo de color amarillo translúcido $(2 \mathrm{ml}$ aproximadamente por tubo evitando aspirar la fracción de células sanguíneas rojas) y se procedió a irrigar el saco periodontal tratado teniendo la precaución de depositar el concentrado lo más apical posible (Fig. 3). La paciente consultó a los 45 días por reagudización del proceso, planificándose un segundo acceso quirúrgico; esta vez, asumiendo la incompleta eliminación de los depósitos duros por vía convencional. Se elevó colgajo mucoperióstico, se eliminó el tejido de granulación, se realizó el pulido radicular de rigor (Fig. 4a) y se rellenó el defecto con membranas de L-PRF (Fig. 4b). Se citó a control a los 7 - 14 - 30 días, 3 y 6 meses.
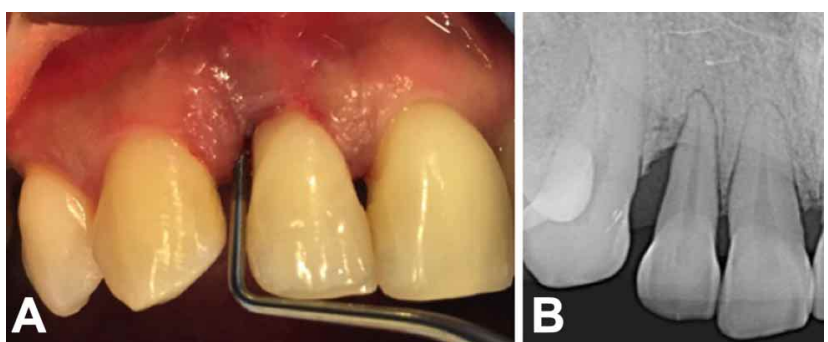

Fig. 1. Se puede observar la presencia de un saco periodontal de $10 \mathrm{~mm}$ de profundidad, tanto al examen clínico (A); como radiológico $(\mathrm{B})$.

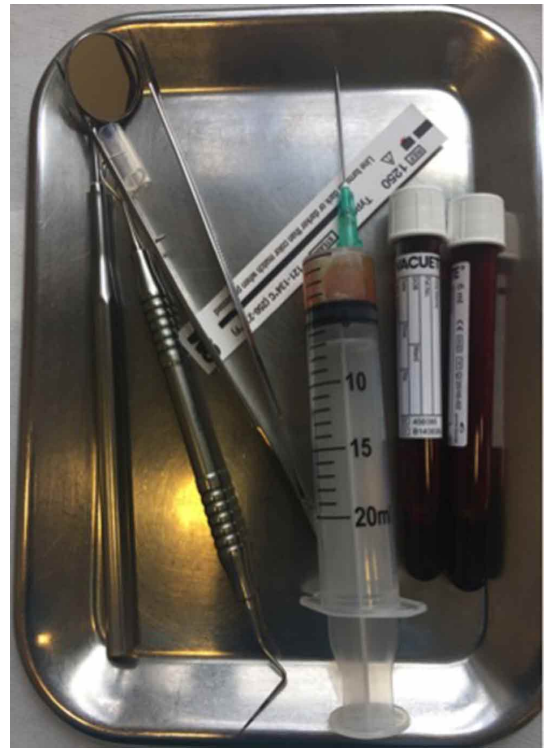

Fig. 2. Elementos necesarios para toma de muestras para i-PRF (mediante punción venosa de la vena cubital), tomando $5 \mathrm{ml}$ en 2 tubos Vaccuette.

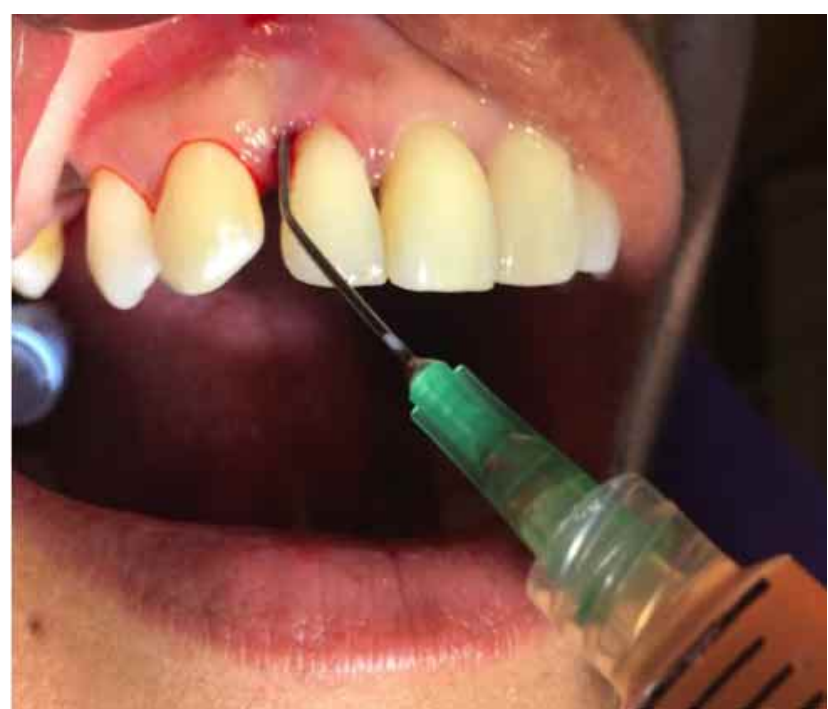

Fig. 3. Irrigación del saco periodontal tratado teniendo la precaución de depositar el concentrado lo más apical posible.
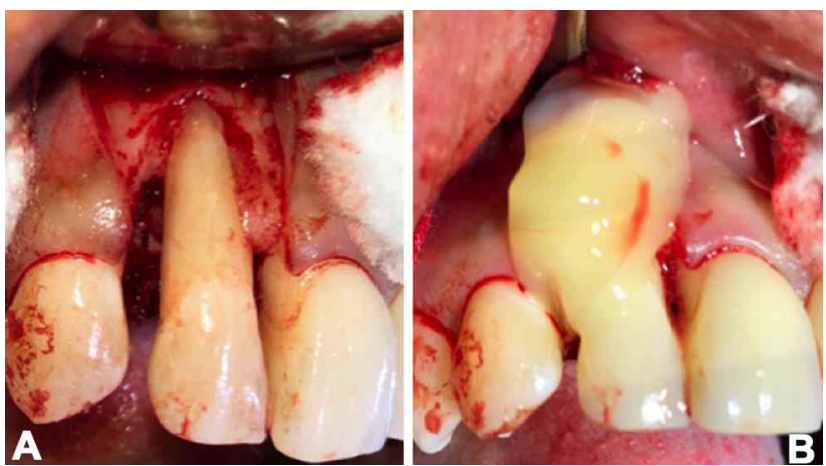

Fig. 4. Elevación de un colgajo mucoperióstico, eliminación del tejido de granulación, y pulido radicular $(A)$. Relleno del defecto con membranas de L-PRF (B). 
Caso clínico 2. Paciente sexo femenino de 84 años de edad, sin antecedentes mórbidos. Consultó por aumento de volumen y sangramiento en relación a proximal de dientes 1.1 y 2.1 . Al examen clínico se observó diente 2.1 vital, oclusión normal en MIC e interferencia en protrusión y saco periodontal de $9 \mathrm{~mm}$ de profundidad por mesial (Fig. 5a). En la radiografía periapical se verificó un defecto infra óseo en la zona examinada (Fig. 5b). Se alivió la oclusión en protrusión y se repitió procedimiento descrito para el caso anterior. Se citó a la paciente a control clínico y radiográfico al mes, 3 y 6 meses.

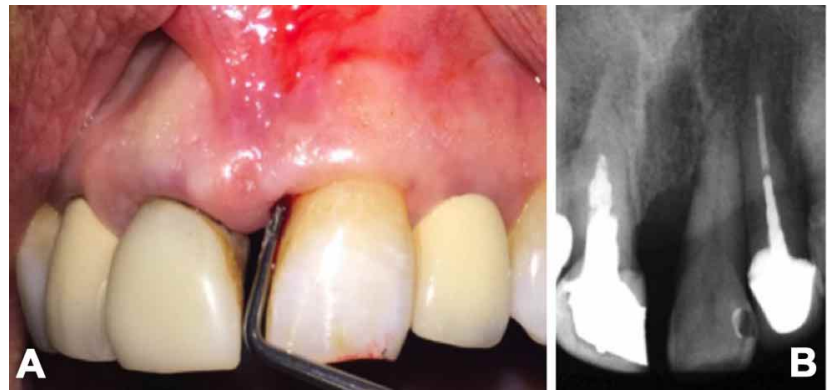

Fig. 5. Se observa diente 2.1 vital, oclusión normal en MIC e interferencia en protrusión y saco periodontal de $9 \mathrm{~mm}$ de profundidad por mesial (A). Se observa defecto infra óseo en la zona examinada en la radiografía periapical (B).

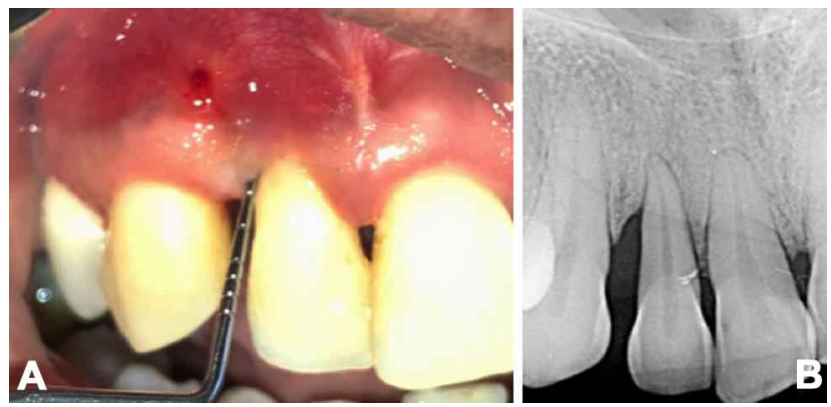

Fig. 6. Caso 1. Control clínico y radiográfico 6 meses.

\section{DISCUSIÓN}

Las secuelas de la enfermedad periodontal en términos del desafío que representan para el clínico son sin lugar a dudas un territorio propicio para la aplicación de técnicas regenerativas tanto para tejidos duros y/o blandos. Los concentrados plaquetarios aportan factores de crecimiento en cantidades "suprafisiológicas" que potencialmente serían responsables de sobre regular el proceso preparativo en beneficio del paciente (Varela et al.). En ambos casos presentados pudimos observar un efecto positivo del i-PRF en la cicatrización del saco periodontal tratado, en el caso 2, incluso esbozándose un subjetivo cambio en la radiografía periapical de con-

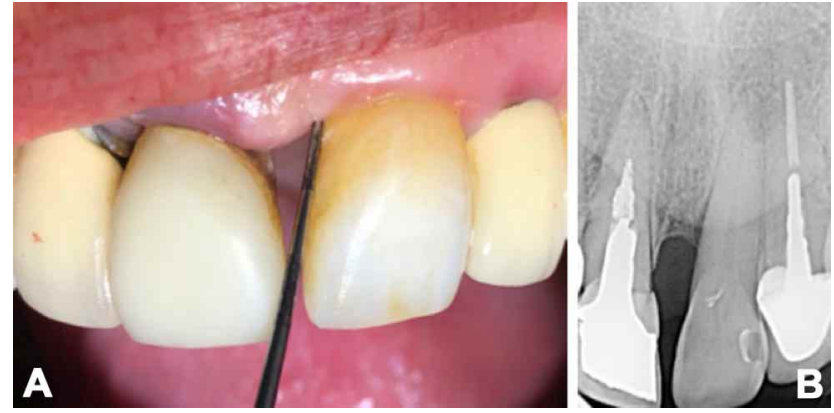

Fig. 7. Caso 2. Control clínico y radiográfico 6 meses.

trol a los 6 meses (Fig. 7), prematuro para este tipo de situaciones caracterizada por la ausencia de evidencias macroscópicas radiográficas antes de los 9-12 meses. En el caso 1 nos encontramos con un reactivación del absceso periodontal original atribuido teóricamente a la remoción incompleta de los depósitos duros de la superficie radicular debido a la falta de acceso y visibilidad para este tipo de defectos por su profundidad (Herrera et al., 2000), por lo que fue necesario complementar el pulido radicular con irrigación de i-PRF con un acceso quirúrgico más aplicación de membranas de L-PRF, logrando así la remisión de la infección, evidenciada en el control clínico y radiográfico a los 6 meses (Fig. 6). El uso de estas membranas se describe en la literatura con positivos resultados, no así el uso del iPRF, terreno sin evidencia clínica disponible a la fecha (Castro et al., 2017a).

Algunos autores reportan un efecto negativo de la edad en el resultado del concentrado obtenido en términos de concentración de citoquinas (Bai et al., 2018), pero de todas maneras contribuye a un mejor escenario en donde la reparación se lleva a cabo, situación del caso 2. La versión inyectable del L-PRF posee una versatilidad que la membrana no tiene, lo que permite su uso como irrigación del saco periodontal, reemplazando a la tradicional clorhexidina, con propiedades bactericidas conocidas sobre los patógenos periodontales, pero también con efectos citotóxicos sobre fibroblastos y células endoteliales, por mencionar algunas células del huésped afectadas (Fabbro et al., 2016). De hecho, existe un protocolo de preparación e indicaciones del L-PRF para diferentes situaciones clínicas en el cual se prohíbe el uso de la clorhexidina durante las primeras 48 horas post-cirugía para no interferir en la función de los fibroblastos (Pinto et al.). Existe evidencia con base en una revisión sistemática, del efecto antimicrobiano de los concentrados plaquetarios en general. Cabe consignar que los estudios primarios fueron principalmente in vitro; y algunos, en animales (Fabbro et al.). También existe evidencia 
de la actividad antimicrobiana para patógenos periodontales; sin embargo queda pendiente dilucidar el espectro y mecanismo de acción (Yang et al., 2015).

Al control de los 6 meses podemos observar remisión completa de la infección en el sitio tratado, lo que se manifiesta con ausencia de sangrado al sondaje y una profundidad de $2 \mathrm{~mm}$, compatible con salud periodontal.

Incluso se podrían inferir las mismas aplicaciones descritas en terapias inyectables del PRP para el i-PRF, como por ejemplo en tendinopatías, alopecia, intraarticularmente en lesiones de cartílago (Marques et al., 2015) e incluso en el tratamiento de la osteoartritis de la articulación temporomandibular (Bousnaki et al., 2018) agregando el beneficio adicional para el i-PRF de la ausencia de anticoagulantes y compuestos químicos como el cloruro de calcio, siendo requisito previo el demostrar en modelos animales la validez y confiabilidad de la técnica.

CORREA, A. J.; ALISTER, J. P. \& MANTEROLA, C. Use of injectable Platelet-Rich Fibrin (i-PRF) in infra osseous defects in non-surgical periodontal therapy. Report of two cases. Int. J. Odontostomat., 13(3):271-274, 2019.

ABSTRACT: Second generation platelet concentrates include the use of injectable platelet-rich fibrin (i-PRF), which has generated increasing interest because it is derived from immediate centrifugation of venous blood from the patients themselves. It provides high concentrations of vascular endothelial growth factor, transforming growth factor beta, and platelet-derived growth factor, among other proteins that initiate and coordinate the healing process. Its null cytotoxicity and liquid consistency has opened new research lines in the field of oral surgery and periodontics, as an irrigation substance. The aim of this manuscript was to report the use of i-PRF, as a subgingival irrigator in conventional periodontal treatment of infra osseous defects, with six months follow-up. In both cases, a positive effect of irrigation was confirmed. These findings, open the debate as regards the use of traditional pharmaceutical products (such as chlorhexidine), versus autonomous preparations without adverse effects reported to date.

KEY WORDS: periodontics/therapy, alveolar bone loss, alveolar bone loss/therapy, platelet-rich fibrin i-PRF, therapeutic irrigation, leukocyte- and platelet-rich fibrin.

\section{REFERENCIAS BIBLIOGRÁFICAS}

Bai, M. Y.; Chuang, M. H.; Lin, M. F.; Tang, S. L.; Wong, C. C. \& Chan, W. P. Relationships of age and sex with cytokine content and distribution in human platelet fibrin gels. Sci. Rep., 8(1):10642, 2018.
Bousnaki, M.; Bakopoulou, A. \& Koidis, P. Platelet-rich plasma for the therapeutic management of temporomandibular joint disorders: a systematic review. Int. J. Oral Maxillofac. Surg., 47(2):188-98, 2018.

Castro, A. B.; Meschi, N.; Temmerman, A.; Pinto, N.; Lambrechts, P.; Teughels, W. \& Quirynen, M. Regenerative potential of leucocyteand platelet-rich fibrin. Part A: intra-bony defects, furcation defects and periodontal plastic surgery. A systematic review and metaanalysis. J. Clin. Periodontol., 44(1):67-82, 2017a.

Castro, A. B.; Meschi, N.; Temmerman, A.; Pinto, N.; Lambrechts, P.; Teughels, W. \& Quirynen, M. Regenerative potential of leucocyteand platelet-rich fibrin. Part B: sinus floor elevation, alveolar ridge preservation and implant therapy. A systematic review. J. Clin. Periodontol., 44(2):225-34, 2017b.

Cortellini, S.; Castro, A. B.; Temmerman, A.; Van Dessel, J.; Pinto, N.; Jacobs, R. \& Quirynen, M. Leucocyte- and platelet-rich fibrin block for bone augmentation procedure: A proof-of-concept study. J. Clin. Periodontol., 45(5):624-34, 2018

Fabbro, M. D.; Bortolin, M.; Taschieri, S.; Ceci, C. \& Weinstein, R. L. Antimicrobial properties of platelet-rich preparations. A systematic review of the current pre-clinical evidence. Platelets, 27(4):27685,2016

Herrera, D.; Roldán, S.; González, I. \& Sanz, M. The periodontal abscess (I). Clinical and microbiological findings. J. Clin. Periodontol., 27(6):387-94, 2000

Marques, L. F.; Stessuk, T.; Camargo, I. C.; Sabeh Junior, N.; dos Santos, L. \& Ribeiro-Paes, J. T. Platelet-rich plasma (PRP): methodological aspects and clinical applications. Platelets, 26(2):101-13, 2015.

Pinto, N. R.; Ubilla, M.; Zamora, Y.; Del Rio, V.; Dohan Ehrenfest, D. M. \& Quirynen, M. Leucocyte- and platelet-rich fibrin (L-PRF) as a regenerative medicine strategy for the treatment of refractory leg ulcers: a prospective cohort study. Platelets, 29(5):468-75, 2018.

Sohn, D. S. Lecture Titled with Sinus and Ridge Augmentation with CGF and AFG. Tokyo, Symposium on CGF and AFG, 2010.

Tonetti, M. S.; Jepsen, S.; Jin, L. \& Otomo-Corgel, J. Impact of the global burden of periodontal diseases on health, nutrition and wellbeing of mankind: A call for global action. J. Clin. Periodontol., 44(5):456-62, 2017.

Varela, H. A.; Souza, J. C. M.; Nascimento, R. M.; Araújo, R. F. Jr.; Vasconcelos, R. C.; Cavalcante, R. S.; Guedes, P. M. \& Araújo, A. A. Injectable platelet rich fibrin: cell content, morphological, and protein characterization. Clin. Oral Investig., 23(3):1309-18, 2018.

Yang, L. C.; Hu, S. W.; Yan, M.; Yang, J. J.; Tsou, S. H. \& Lin, Y. Y. Antimicrobial activity of platelet-rich plasma and other plasma preparations against periodontal pathogens. J. Periodontol., 86(2):310-8, 2015

Dirección para correspondencia:

Dr. Carlos Manterola

Departmento de Cirugía

Universidad de La Frontera

CEMyQ

Manuel Montt 112, Of. 408

Temuco - CHILE

E-mail: carlos.manterola@ufrontera.cl

Received: 03-01-2019

Accepted: 24-04-2019 